\title{
Vacuum Sealed Plastic Container Closure
}

National Cancer Institute

\section{Source}

National Cancer Institute. Vacuum Sealed Plastic Container Closure. NCI Thesaurus. Code C96121.

Plastic closures used on packages where the pressure inside the package is less than atmospheric. 